\title{
Laboratorios virtuales: una alternativa para mejorar el rendimiento de los estudiantes y la optimización de recursos económicos
}

\section{Virtual laboratories: an alternative to improve student performance and optimize economic resources}

Ing. Jorge Encalada Noboa, Msc.

Universidad de Guayaquil, Ecuador

Ing. Christian Pavón Brito, Msc.

Universidad de Guayaquil, Ecuador

Autor para correspondencia: christian.pavonb@ug.edu.ec, jorge.encaladan@ug.edu.ec

Fecha de recepción: 10 de Septiembre de 2016 - Fecha de aceptación: 01 de Noviembre de 2016

Resumen: En enseñanza de las Ciencias las actividades de laboratorio aumentan el interés de los estudiantes, por lo que favorece su aprendizaje. Sin embargo, debido a la falta de laboratorios en las instituciones educativas, o a su vez, insuficiente instrumentación en los laboratorios, las prácticas de laboratorio rara vez se realizan en las escuelas y colegios del país. No obstante, debido a los resultados del vertiginoso desarrollo en las tecnologías de la información y la comunicación, estos experimentos pueden realizarse de una forma rápida y con resultados similares o mejores de los que se obtiene con laboratorios bien equipados. En este estudio se presenta el uso de simulaciones virtuales relacionadas con las unidades de primer año del Bachillerato General Unificado del Ecuador con estudiantes del año antes mencionado y se analizan sus efectos en el rendimiento académico de los mismos. Para este fin, se realizó una intervención con seis experimentos virtuales utilizados en el grupo experimental. El resultado de este estudio mostró que las aplicaciones de laboratorio virtuales tuvieron efectos positivos sobre el rendimiento académico de los estudiantes, en comparación con los métodos tradicionales de enseñanza.

Palabras claves: laboratorio virtual; animaciones de ordenador; enseñanza de ciencias; prácticas de laboratorio

Abstract: In teaching science the laboratory activities increase students interest, so that favors learning. However, due to the lack of laboratories in educational institutions, or in turn, inadequate instrumentation in laboratories, laboratory practices are rarely performed in schools and colleges in the country. Nevertheless, due to the results of the rapid development in information and communications technology, these experiments can be performed quickly and with similar or better results than those obtained with well-equipped laboratories. In this study it is presented the use of virtual simulations related to the units of the first year of the General Unified Baccalaureate of Ecuador with students of the aforementioned year and its effects are analyzed in academic achievement thereof. To this end, was performed an intervention with six virtual experiments used in the experimental group. The result of this study showed that virtual laboratory applications had positive effects on academic performance of students, compared with traditional teaching methods. Key words: virtual laboratory; computer animations; science teaching; lab practices 


\section{Introducción}

El vertiginoso desarrollo del Internet junto con los avances en las tecnologías de la comunicación y la información han dado paso para disponer de todas las condiciones previas necesarias para la adopción de este poderoso medio en el ámbito educacional.

En cuanto al campo tecnológico, el desarrollo de animaciones multimedia han sido ampliamente propuestos y reconocidos como un importante avance tecnológico para el apoyo a la educación. Una de las capacidades únicas de esta tecnología es el éxito de la traducción de los conceptos abstractos en eventos visualizados junto con la posibilidad de interacción de los usuarios con ellos, que en la vida real podría ser limitado debido a la distancia, el tiempo y los factores de seguridad (Alexiou et al., 2004).

Las funcionalidades que un entorno virtual podría proporcionar, en combinación con el creciente interés en la investigación para el aprendizaje a distancia, llevaron a la creación de una amplia gama de aplicaciones que adoptan la tecnología de simulaciones con el fin de apoyar el proceso de aprendizaje y de conformación virtual basadas en ambientes de aprendizaje (Blázquez, 2001). Sin embargo, aparte de los aspectos tecnológicos que abrazan las simulaciones, están los aspectos educativos, por ejemplo, los contenidos de aprendizaje, así como el modelo educativo que se debe utilizar.

Una de las tendencias más en auge es la del aprendizaje experimental o aprendizaje por la experiencia, donde los usuarios se encuentran como actores y no como receptores pasivos de la información proporcionada. El aprendizaje experimental implica un alto grado de interactividad de los usuarios, tanto con otros usuarios, así como con los objetos disponibles en el ambiente virtual.

La evolución notada en las tecnologías de la información también ha afectado a Ecuador, tanto en el sector industrial, así como en el campo educativo. En lo que respecta al campo de la educación, se debe mencionar que en la última década, la mayoría de las escuelas secundarias han sido equipadas con laboratorios de computación con el fin que los estudiantes se familiaricen con la sociedad de la información y las nuevas tecnologías (Unesco, 2005).

Sin embargo, el sistema educativo no ha aprovechado al máximo las capacidades adicionales que podrían presentar los laboratorios de computación de la escuela. Por lo tanto, este estudio enfatiza el uso de simulaciones virtuales y muestra los resultados obtenidos luego de realizar una intervención con estudiantes de una Unidad Educativa de la ciudad.

\section{Metodología}

\section{$\underline{\text { Sujetos }}$}

Los sujetos fueron 50 estudiantes, entre los cuales se cuentan 25 hombres y 25 mujeres. Todos estaban cursando el Primer Año de Bachillerato General Unificado del Ecuador en una institución educativa de la ciudad de Guayaquil. La edad de ellos está comprendida entre los 14 y 15 años de edad. 


\section{Tareas y materiales instruccionales}

Las dos tareas instruccionales corresponden a las unidades de El movimiento de los cuerpos en una dimensión y Trabajo, energía y potencia (Ministerio de Educación del Ecuador, 2011) y los temas tratados fueron los conceptos de gráficas de posición, velocidad y aceleración y la ley de la conservación de energía.

\section{Instrumentos}

El instrumento para medir el desempeño de los estudiantes fue una prueba de opciones múltiples que consta de 40 ítems.

\section{$\underline{\text { Procedimiento }}$}

El procedimiento seguido durante la intervención fue el siguiente: (1) Presentar al grupo experimental la intervención y al grupo de control impartir la clase de acuerdo al modelo tradicional. El contenido y los problemas propuestos fueron los mismos para ambos grupos, lo único que vario fue la manera de presentarlos. (2) Administrar la prueba de conocimientos tanto al grupo experimental como de control. La prueba de conocimientos fue la misma para ambos grupos.

\section{$\underline{\text { Hipótesis }}$}

La hipótesis de investigación es: Aquellos estudiantes a los cuales se aplica la intervención tienen mejor desempeño que aquellos estudiantes a los cuales no se aplica la intervención.

La hipótesis nula es: Aquellos estudiantes a los cuales se aplica la intervención tienen el mismo desempeño de aquellos estudiantes a los cuales no se aplica la intervención.

\section{$\underline{\text { Análisis de datos }}$}

El análisis estadístico aplicado en esta investigación fue la prueba t de Student pareada con un nivel de significación $\mathrm{p}<0,05$.

\section{Resultados}

En la Figura 1 se muestran los resultados de la prueba de desempeño administrada al grupo experimental y de control. 


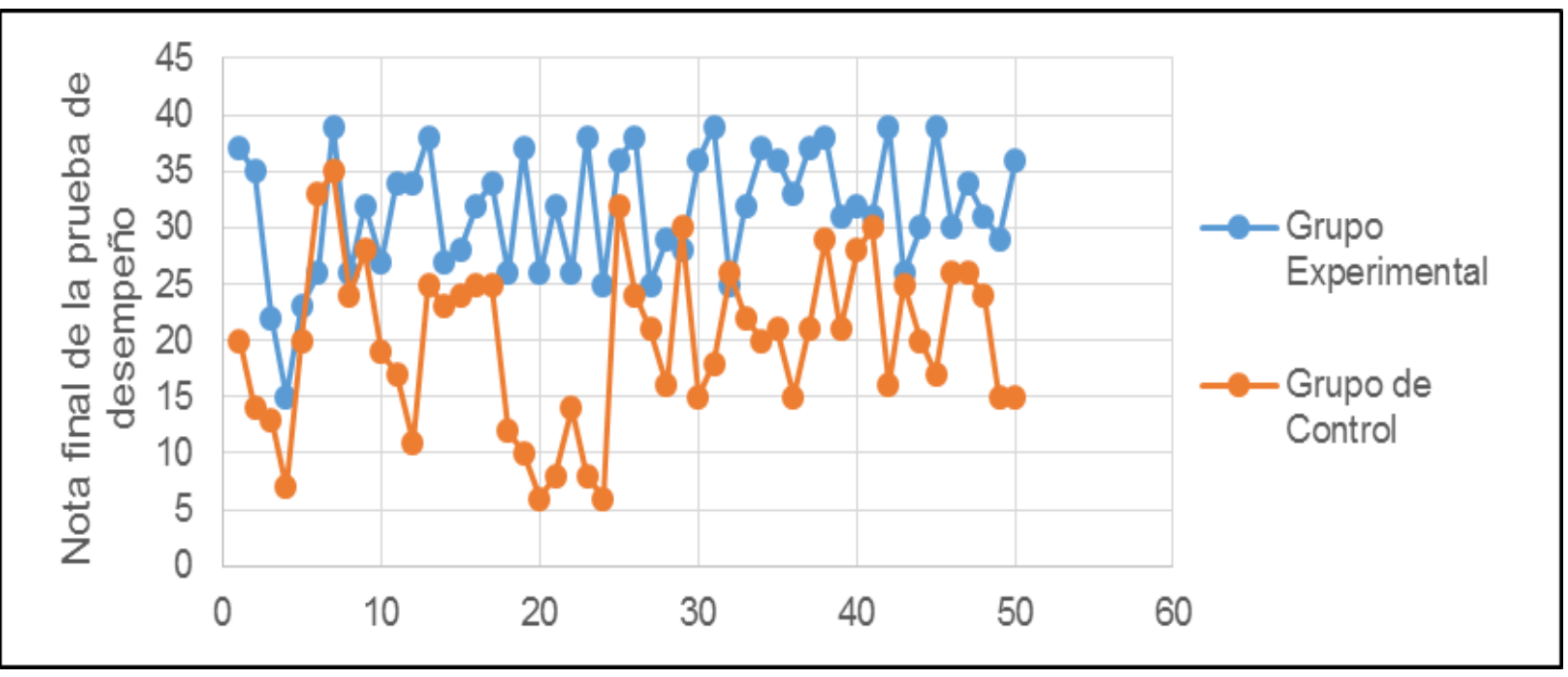

Figura 1. Resultados individuales de la prueba de desempeño

La prueba t de Student pareada dio un valor de $\mathrm{p}=0,0068$ por lo tanto se rechaza la hipótesis nula y se acepta la hipótesis de investigación. A continuación, en la Tabla 1, se muestra un resumen de los resultados de la prueba $t$.

Tabla 1. Datos estadísticos de la prueba de desempeño

\begin{tabular}{ll}
\hline Valor de P (doble cola) & $<0,0001$ \\
\hline Nivel de significancia $(<0.05)$ & Extremadamente significante \\
Media del Grupo A menos el Grupo B & $-11,52$ \\
Intervalo de confianza (desde -12.81 hasta -9.19) & $95 \%$ \\
t & 8,94 \\
Grados de libertad & 90,82 \\
\hline
\end{tabular}

El valor de t obtenido fue de 8,94. El valor de $t_{\text {crítico }}$ para 90,82 grados de libertad, con un nivel de confianza de 0.005 es de 1,66 (Triola, 2009). En este caso, como 8,94 es mayor que 1,66 se rechaza la hipótesis nula y se acepta la hipótesis de investigación.

\section{Conclusiones}

A medida que el internet se convierte en una verdadera red multifunciones con un aumento constante de ancho de banda y la disminución en el tiempo de respuesta, el entorno se vuelve más adecuado para iniciativas tales como laboratorios virtuales.

Tradicionalmente, el laboratorio ha sido un componente integral de la educación para relacionar los conceptos fundamentales de los fenómenos básicos con la vida real. Llevado a 
cabo en gran medida dentro del formato convencional, el laboratorio implica el uso de experimentos en su mayoría predeterminados que simulan fenómenos básicos que se encuentran en situaciones del mundo real. Por desgracia, el sistema de aula-laboratorio implica la necesidad de grandes cantidades de espacio y a veces una complicada la logística para crear y mantener un ambiente de aprendizaje apropiado.

Sin embargo, actualmente se está cuestionando el papel de los laboratorios convencionales para la formación y la comprensión de lo problemas educativos. Los avances de la tecnología de la información y la comunicación han dado lugar a algunos procesos de aprendizaje siendo replicadas en diversos grados, en forma de cursos disponibles a través de internet, superando de esta manera algunas limitaciones del sistema de conferencia-aulalaboratorio tradicional. Los laboratorios virtuales tienen las siguientes ventajas con respecto a los laboratorios reales:

- El procedimiento de aprendizaje a través de laboratorios reales en algunas ocasiones es peligroso.

- Algunos experimentos no se pueden realizar debido a limitaciones de tiempo o riesgos.

- El costo de construir y dar mantenimiento a un laboratorio real no se ajusta a los presupuestos de la institución educativa.

- Hay experimentos que se pueden simular sólo en ordenadores.

Las simulaciones virtuales proporcionan una representación amigable de la información proporcionada, la interacción con el sistema, que no requiere conocimientos avanzados de manejo de software junto con la reducción de costos en comparación con otras tecnologías. Además, uno de los aspectos más importantes de esta propuesta es el hecho de que el desarrollo de Laboratorios Virtuales puede ser totalmente compatible con los Laboratorios de Computación existentes, sin la necesidad de inversiones e infraestructuras adicionales.

\section{Bibliografía}

Alexiou, A., Bouras, C., Giannaka, E., Kapoulas, V., Nani, M., Tsiatsos, T. (2004). Using VR technology to Support e-Learning. The 3D Virtual Radiopharmacy Laboratory: Proceedings of the 6th International Workshop on Multimedia Network Systems and Applications. Tokyo, Japan, $268-273$.

Blázquez, F. (2001). Sociedad de la Información y Ecucación. Mérida, España: Junta de Extremadura.

Ministerio de Educación del Ecuador (2011). Lineamientos Curriculares para el Bachillerato General Unificado: Física - Primer Curso. Quito, Ecuador: Ministerio de Educación del Ecuador.

Triola, M. (2009). Estadística. México DF, México: Pearson. 
Unesco (2005). Las tecnologías de la información y la comunicación en la enseñanza. Montevideo, Uruguay: Gráficas Don Bosco. 